\title{
Long-term expansion enhances the expression of tumor suppressor genes in human bone marrow-derived mesenchymal stem cells
}

\author{
Loan Thi Tung Dang ${ }^{1}$, Anh Thi Van Bui ${ }^{2}$, Nhat Chau Truong ${ }^{2}$, Huy Duc Van ${ }^{2}$, Phuc Van Pham ${ }^{1,2, *(1)}$
}

${ }^{1}$ Laboratory of Stem Cell Research and Application, VNUHCM University of Science, Ho Chi Minh City, Viet Nam

${ }^{2}$ Stem Cell Institute, VNUHCM University of Science, Ho Chi Minh City, Viet Nam

\section{Correspondence}

Phuc Van Pham, Laboratory of Stem Cell Research and Application, VNUHCM University of Science, Ho Chi Minh City, Viet Nam

Stem Cell Institute, VNUHCM University of Science, Ho Chi Minh City, Viet Nam

Email: pvphuc@hcmuns.edu.vn

History

- Received: 15 February 2019

- Accepted: 17 March 2019

- Published: 22 March 2019

DOI :

https://doi.org/10.32508/stdj.v22i1.1661

\section{Check for updates}

\section{Copyright}

(c) VNU-HCM Press. This is an openaccess article distributed under the terms of the Creative Commons Attribution 4.0 International license.

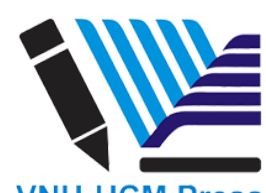

\begin{abstract}
Introduction: Mesenchymal stem cells (MSCs) are possibly the most potent type of stem cells for the treatment of many diseases since they possess many advantageous properties, such as abundant source, ease of isolation, and potential to differentiate and trans-differentiate into different types of cells. Although the therapeutic potential of expanded MSCs has been well proven, their biosafety features have not been fully understood. This study aimed to investigate some changes in phenotype and gene expression of bone marrow derived MSCs after long term expansion. Methods: In this study, expanded mesenchymal stem cells derived from human bone marrow (hBMSCs) were identified for their characteristics (which included morphology, immunophenotype, and differentiation potential) at passages 5, 10 and 15. Moreover, they were evaluated for the expression of various tumor suppressor genes (PTEN, p16, and p53) by real-time RT-PCR. Results: The results showed that the hBMSCs at passage 15 displayed a change in morphology and a slight reduction of the expression of CD44 and CD90, whereas their potential for adipogenic and osteogenic differentiation was maintained. Moreover, the expression of tumor suppressor genes in the hBMSCs increased after long-term culture. Conclusion: It could be assumed that prolonged cultures of more than 15 passages drove the hBMSCs into senescence phase. Cultured hBMSCs below passage 10 seemed to be more effective in application because their properties were still preserved. Key words: Bone marrow-derived mesenchymal stem cells, Long-term expansion, Tumor suppressor genes, Stem cell aging
\end{abstract}

\section{INTRODUCTION}

There have been a remarkable number of research studies and clinical trials of mesenchymal stem cells (MSCs) in recent years. MSCs have shown therapeutic effects for several diseases, including graft versus host disease (GVHD), heart failure, chronic spinal cord injury ${ }^{1}$, diabetes mellitus ${ }^{2,3}$, and even cancer ${ }^{4}$. While MSC-based therapy represents a potentially valuable and potent application, the biosafety of MSCs, particularly of expanded MSCs, has not been well-studied. Long-term in vitro cultured MSCs are assumed to enter a senescence phase after approximately 20 doubling populations ${ }^{5}$. They may bypass the senescence phase and continue to divide until they enter the crisis phase, followed by cell death ${ }^{6}$. Some of the MSCs may bypass the crisis phase and become transformed cell lines ${ }^{5}$.

Phenotypic and cytogenetic methods are used to evaluate cell senescence and transformation; moreover, changes in gene expression and DNA methylation are also employed ${ }^{7}$. Transformation of human cells is assumed to be associated with gene expression changes, particularly of genes involved in telomerase activity, tumor suppressor genes, and those related to activation of oncogenes ${ }^{8}$. Long-term expansion of human MSCs is thought to decrease therapeutic efficacy due to the reduction of the MSC properties ${ }^{7,9}$.

To identify the alteration of human bone marrowderived MSCs (hBMSCs) after prolonged culture, this study evaluated the changes in MSC characteristics, including morphology, the potential of mesoderm lineage differentiation, and the profile of MSC surface markers as well as expression of certain tumor suppressor genes ( $p 16, p 53$, and PTEN). This study will provide further knowledge in the understanding of the properties of long-term cultured MSCs. The findings will impact the basis of cultured MSCs in research and clinical applications.

\section{METHODS}

\section{Cell cultures}

The human bone marrow-derived mesenchymal stem cells (hBMSCs) were provided by the Laboratory of Stem Cell Research and Application, VNUHCM University of Science. The cells were cultured according mesenchymal stem cells. Sci. Tech. Dev. J.; 22(1):136-142. 
to a previously published protocol from the laboratory ${ }^{10,11}$. Briefly, the cells were thawed from liquid nitrogen $\left(-196^{\circ} \mathrm{C}\right)$ and cultured in MSC Cult medium under the condition of $37^{\circ} \mathrm{C}, 5 \% \mathrm{CO}_{2}$ in an incubator. When confluency was reached, the cells were passaged and continuously cultured in fresh medium. The cultured cells were harvested at passages 5, 10, and 15 for use in experiments.

\section{Flow cytometric analysis of cell surface markers}

The surface markers of the cultured cells were detected by immunostaining using the following monoclonal antibodies: CD14-FITC, CD34-FITC, CD44$\mathrm{PE}$, and CD166-PE (BD Biosciences, San Jose, CA, USA), CD73-FITC, and CD90-FITC (Santa Cruz Biotechnology, Dallas, TX, USA) for 30 minutes. The cells were washed with FACS buffer after that, and then suspended in sheath fluid and analyzed using a FACS Calibur flow cytometer (BD Biosciences) with CELLQuest software (BD Biosciences).

\section{Osteogenic and adipogenic differentiation}

The hBMSCs were induced to differentiate into adipocytes and osteocytes in the adipogenic and osteogenic medium, respectively, for 21 days. The adipogenic medium included low glucose DMEM/F12 supplemented with 10\% FBS (Thermo Fisher Scientific, Waltham, MA, USA), and $1 \%$ antibiotic-antimycotic, $1 \mathrm{mM}$ dexamethasone, 0.5 $\mu \mathrm{M}$ 3-isobutyl-1-methylxathine (IBMX), $200 \mu \mathrm{M}$ indomethacin, and $10 \mathrm{ng} / \mathrm{mL}$ insulin (all reagents were purchased from Sigma-Aldrich, St. Louis, MO, USA). The osteogenic media included low glucose DMEM/F12 supplemented with 10\% FBS, $1 \%$ antibiotic-antimycotic, $50 \mu \mathrm{g} / \mathrm{ml}$ AsAP (apoptosisand splicing-associated protein), $0.1 \mu \mathrm{M}$ dexamethasone, and $100 \mathrm{mM} \beta$ - glycerophosphate (all reagents above were obtained from Sigma-Aldrich).

The cells were plated at $10^{3} \mathrm{cell} / \mathrm{cm}^{2}$ in the induction medium. The medium was changed every 3 days. After 21 days of induction, the cells were stained with Oil Red O or Alizarin Red to identify lipid droplets or mineralized matrix content, respectively.

\section{Real-time RT-PCR}

The expression of tumor suppressor genes, such as p16, p53, and PTEN, were evaluated by quantitative reverse transcription PCR (qRT-PCR). At first, total RNA was isolated from hBMSCs using Easy-BLUE Total RNA Extraction Kit (iNtRON, Republic of Korea) following the manufacturer's protocol. RNA concentration and purity of RNA were determined by the BioPhotometer Plus system (Eppendorf, Hamburg, Germany). Then, RNA levels were evaluated by LightCycler480II machine (Roche, Basel, Switzerland) using qPCRBIO SyGreen 1-Step Lo-ROX Kit (PCR Biosystems, UK). The mixtures were set at $45^{\circ} \mathrm{C}$ for 10 minutes in reverse transcription reaction and then heated at $95^{\circ} \mathrm{C}$ for 2 minutes. Amplification was performed for 40 cycles of $95^{\circ} \mathrm{C}$ for 5 seconds, followed by $60^{\circ} \mathrm{C}$ for 20 seconds. GAPDH was used as an internal standard and the relative expression of selected genes was determined using the $\Delta \Delta \mathrm{Cq}$ method.

\section{Data Analysis}

GraphPad Prism 6 (GraphPad Software, Inc., La Jolla, CA, USA) was used to analyze the data. Statistical significance was defined as $\mathrm{P}<0.05$, and results were analyzed by Student's $t$-test and one-way ANOVA.

\section{RESULTS}

\section{Characterization of hBMSCs}

The MSCs from human bone marrow were thawed and cultured in fresh medium in a $37^{\circ} \mathrm{C}, 5 \% \mathrm{CO}_{2}$ incubator. The cultured adherent cells were let to proliferate until passages 5,10 , or 15 . Then, the cells were characterized for their MSC properties, such as morphology, expression of typical surface markers, and potential for differentiation into mesodermal lineage cells.

The hBMSCs at passage 5 displayed the typical fibroblast shape, which became gradually smoothened after long-term culture or expansion (passages 10 and 15). At the late passage (P15), the border of cultured cells was observed to be different (Figure 1).

Flow cytometry (FCM) analysis demonstrated that the hBMSCs at all three passages expressed the typical MSC markers, including CD44, CD73 and CD90, with greater than $80 \%$ of the cells expressing these markers. Conversely, less than $10 \%$ of the cells expressed the hematopoietic cell markers, CD14 and CD34. Moreover, the results of the differentiation assays demonstrated that hBMSCs maintained their potential of osteogenic and adipogenic differentiation after long-term expansion (passages 10 and 15).

\section{Changes in expression of the various tumor suppressor genes}

To identify the changes in the expression of tumor suppressor genes in long-term cultures of MSCs, we analyzed $p 16, p 53$, and PTEN. In the quantitative RTPCR results, we found an increased expression of $p 16$, 

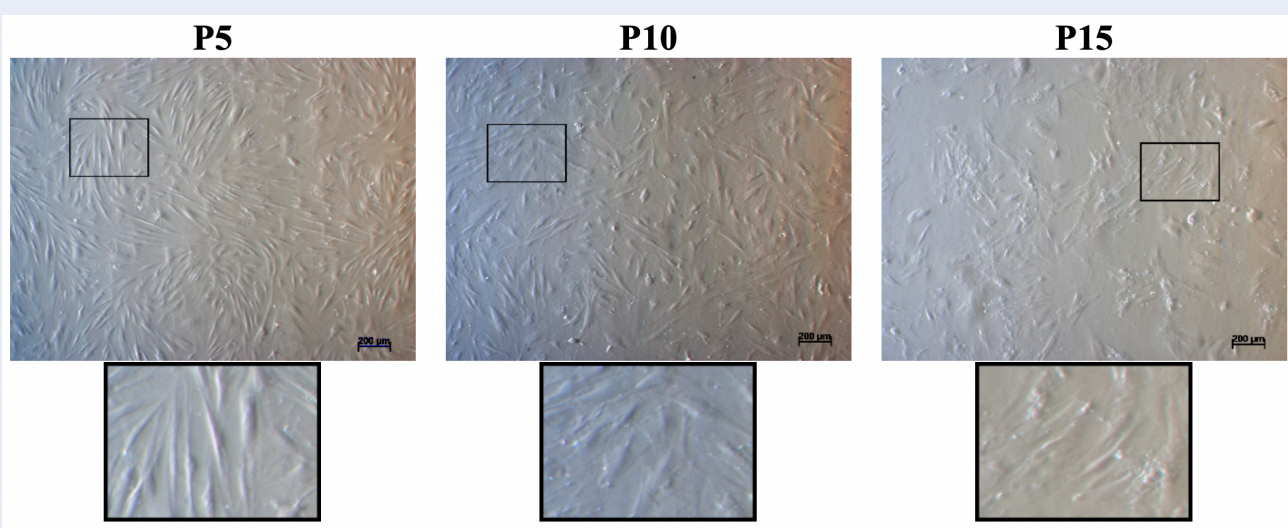

Figure 1: Representative images of cultured hBMSCs with altered morphology at passages 5, 10 and 15. Scale bar $=200 \mu \mathrm{m}$.

p53, and PTEN in hBMSCs at passage 15. Moreover, p16 and PTEN were expressed at passage 15; the hBMSCs were approximately 4 -fold greater than those from passage 5 (hBMSCs), whereas $p 53$ was upregulated more than 11-fold. Meanwhile, there was no change in the expression of $p 16, p 53$ and PTEN levels between hBMSCs at passages 5 and 10 .

\section{DISCUSSION}

Bone marrow-derived mesenchymal stem cells are promising multipotent cells that are being widely used in many clinical applications, such as for the treatment of degenerative tissues, graft-versus-host disease, and autoimmune diseases ${ }^{12}$. For clinical applications, the human MSCs are isolated, culture and expanded ex vivo to obtain a large number of cells. According to the International Society for Stem Cell Research (ISSCR), the expanded MSCs should be checked for their biosafety and effectiveness prior to use in clinical trials. These issues are related to the integrity of the MSCs, including their "stem cell" characteristics, chromosomal stabilities, and their status in in vitro expansion. In this study, we evaluated the changes in hBMSCs with regards to their morphology, immunophenotype, the potential for adipogenic and osteogenic differentiation, and expression of tumor suppressor genes $p 16, p 53$ and PTEN.

It was found that the hBMSCs had the typical morphology of spindle-shaped MSCs at passage 5 and gradually became enlarged and flattened until passage 15 . The altered appearance of hBMSCs suggested that they underwent a senescence phase during the in vitro culture ${ }^{7,13}$. The FACS analysis reinforced that aging hBMSCs had a reduction in the expression of
CD44 and CD90 (95\% and 99\%, respectively, at passage 5 , compared to $81 \%$ and $92 \%$, respectively, at passage 15). The down-regulation of CD90 was documented in cells at the senescence or crisis phase ${ }^{13-15}$. Even when the MSCs transformed, they expressed low levels of CD90 and were negative for CD105 ${ }^{15}$. The CD90- ${ }^{-}$phenotype of transformed MSCs was also described in a previous publication ${ }^{16}$. Furlani described that the transformed or abnormal MSCs, after longterm culture, were devoid of CD44 and CD90 and showed diminished therapeutic effects ${ }^{9}$.

Although our cultured hBMSCs at passage 15 displayed the senescence status, there was a relatively high proportion of hBMSCs (greater than 80\%) expressing positive markers like CD44, CD73, and CD90; a low proportion (about 10\%) of the cells expressed the negative markers, such as CD14 and CD34. The hBMSCs at passages 5, 10 and 15 were able to differentiate into adipocytes and osteocytes. Therefore, the hBMSCs maintained their differentiation potential into mesoderm lineage cells after in vitro prolonged expansion. The same observations were noted in long-term culture of human umbilical cord-derived $\mathrm{MSCs}^{17}$, human bone marrow-derived MSCs ${ }^{15}$, and human adipose-derived MSCs ${ }^{13}$.

The lifespan and status of in vitro long-term cultured MSCs could be predicted by the expression of pluripotent markers, such as OCT4, tumor suppressor genes, such as $p 16^{18}, p 53$, and $P T E N^{8}$, and oncogenes, such as MYC and $R A S^{8}$. P16, $p 53$, and other tumor suppressor genes are assumed to be senescence-related genes; their expression is induced after long-term expansion ${ }^{5,7,19}$. The low $p 16^{I N K 4 A}$ expression together with high OCT4 gene expression represents the robust in vitro proliferation of MSCs ${ }^{18}$. Moreover, the 


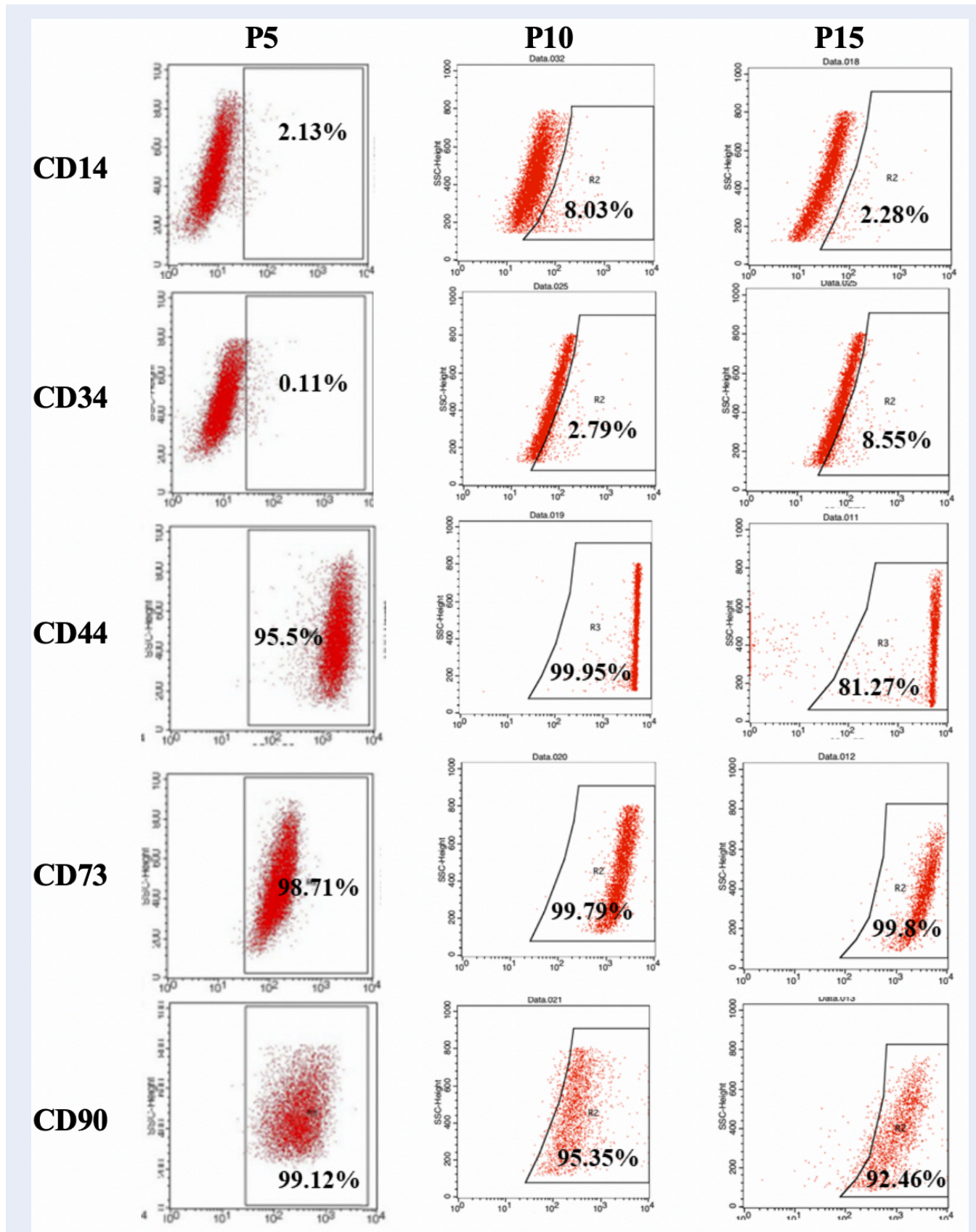

Figure 2: Surface marker expression of hBMSCs at passages 5, 10 and 15. The hBMSCs at the three passages showed a high expression of CD44, CD73, and CD90. On the other hand, CD14 and CD34 were expressed in all passages by about $10 \%$ of the hBMSCs. 

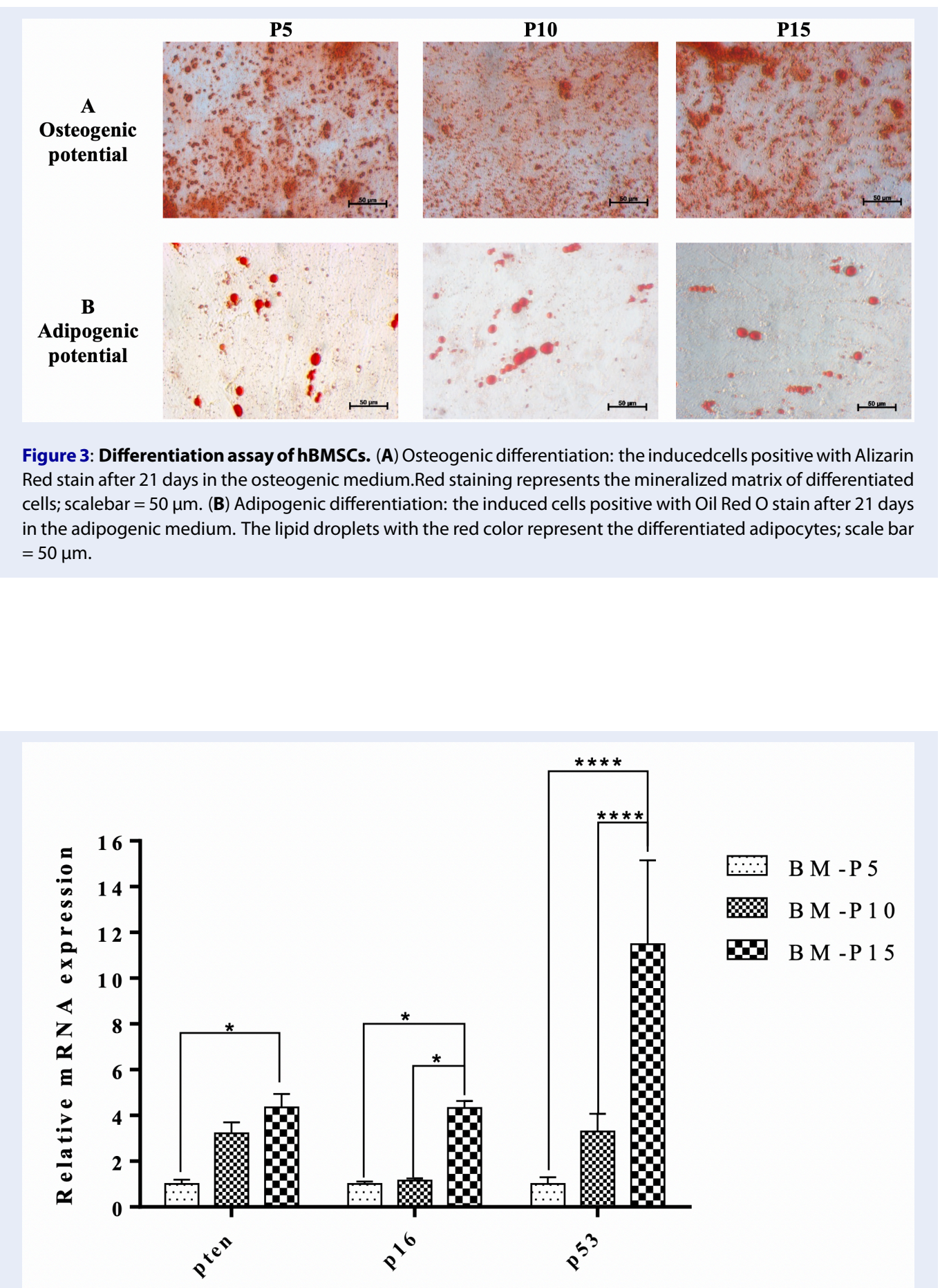

Figure 4: Prolonged cultures enhance the expression of tumor suppressor genes of human bone marrowderived mesenchymal stem cells. Relative expression levels for PTEN, p16, and p53 were assessed using the Livak method. Data shown are comparable to an internal control (GAPDH), with fold change compared to expression levels in hBMSCs at passage 5 (set to 1). Statistical significance was set at $\mathrm{p}<0.05$. 
expression of $p 16$ can be down-regulated so that the MSCs are free from the senescence state in hypoxic conditions or cultures ${ }^{20}$. The loss or depletion of $p 53$ can induce tumorigenesis ${ }^{21}$ or generate mutated and spontaneously transformed hBMSCs ${ }^{12}$, whereas the up-regulation of $p 53$ can induce cell senescence ${ }^{7}$. In our study, the results indicated that the expression of $p 16, P T E N$, and $p 53$ increased 4 - to 11 -fold in hBMSCs at passage 15 , compared to those at passage 5 . The findings concur with the results of altered morphology and reduction of CD90 expression described above.

\section{CONCLUSION}

Our study demonstrated that hBMSCs could display signs of senescence after 15 passages of culture, changes in cell morphology, changes in expression of CD44 and CD90, as well as changes (increase) in the expression of $p 16, p 53$, and PTEN. However, the hBMSCs maintained the potential of adipogenic and osteogenic differentiation. Meanwhile, there was no change in the hBMSCs between passage 5 and passage 10. It is suggested that the expansion of hBMSCs not surpass passage 10 in order to avoid the reduction in quality of the cultured cells. These results require further studies to better understand mesenchymal stem cell biology in vitro to confirm the safety of the stem cells. This will critical prior to applications of the stem cells in clinical treatment or in the development of optimal culture methods for stem cell production for research and application.

\section{ABBREVIATIONS}

FCM: Flow cytometry

GVHD: Graft Versus Host Disease

hBMSCs: human Bone Marrow-derived Stem Cells MSCs: Mesenchymal stem cells

\section{COMPETING INTERESTS}

The authors declare that no competing interests exist.

\section{AUTHORS' CONTRIBUTIONS}

LTTD: designed the study, performed the experiments, analyzed the data and wrote the paper; PVP: designed the study and revised the paper; ATVB, HDV: performed the experiments and analyzed the data; NCT: performed the experiments and reviewed the paper.

\section{ACKNOWLEDGMENTS}

This research was funded by Viet Nam National University, Ho Chi Minh city via project No. C2016-1818 .

\section{REFERENCES}

1. Galipeau J, Sensébé L. Mesenchymal Stromal Cells: Clinical Challenges and Therapeutic Opportunities. Cell Stem Cell. 2018;22(6):824-33. 29859173. Available from: 10.1016/j.stem. 2018.05.004.

2. Cheng SK, Park EY, Pehar A, Rooney AC, Gallicano GI. Current progress of human trials using stem cell therapy as a treatment for diabetes mellitus. Am J Stem Cells. 2016;5(3):74-86. 27853629.

3. El-Badawy A, El-Badri N. Clinical Efficacy of Stem Cell Therapy for Diabetes Mellitus: A Meta-Analysis. PLoS One. 2016;11(4):e0151938. 27073927. Available from: 10.1371/ journal.pone.0151938.

4. Momin EN, Vela G, Zaidi HA, Quiñones-Hinojosa A. The Oncogenic Potential of Mesenchymal Stem Cells in the Treatment of Cancer: Directions for Future Research. Curr Immunol Rev. 2010;6(2):137-48. 20490366. Available from: 10.2174/ 157339510791111718.

5. Rubio D, Garcia S, Paz MF, la Cueva TD, Lopez-Fernandez LA, Lloyd AC, et al. Molecular characterization of spontaneous mesenchymal stem cell transformation. PLoS One. 2008;3(1):e1398. 18167557. Available from: 10.1371/journal. pone.0001398.

6. Stagg J, Pommey S. Properties of Mesenchymal Stem Cells to Consider for Cancer Cell Therapy. In: Dittmar T, Zanker KS, editors. Stem Cell Biology in Health and Disease. Netherlands: Springer; 2010. p. 79-98.

7. Turinetto V, Vitale E, Giachino C. Senescence in Human Mesenchymal Stem Cells: Functional Changes and Implications in Stem Cell-Based Therapy. Int J Mol Sci. 2016;17(7):1164. 27447618. Available from: 10.3390/ijms 17071164 .

8. Takeuchi M, Higashino A, Takeuchi K, Hori Y, Koshiba-Takeuchi $\mathrm{K}$, Makino $\mathrm{H}$, et al. Transcriptional Dynamics of Immortalized Human Mesenchymal Stem Cells during Transformation. PLoS One. 2015;10(5):e0126562. 25978455. Available from: 10.1371/journal.pone.0126562.

9. Furlani D, Li W, Pittermann E, Klopsch C, Wang L, Knopp A, et al. A transformed cell population derived from cultured mesenchymal stem cells has no functional effect after transplantation into the injured heart. Cell Transplant. 2009;18(3):319-31. 19558780. Available from: 10.3727/096368909788534906.

10. Pham VP, Vu BN, Phan LCN, Le MD, Truong CN, Truong HN, et al. Good manufacturing practice-compliant isolation and culture of human adipose-derived stem cells. Biomed Res Ther. 2014;1(4):133-41.

11. Pham PV, Phan NL, Le DM, Le PT, Tran TD, Phan NK. Good manufacturing practice-compliant isolation and culture of human bone marrow mesenchymal stem cells. Prog Stem Cell. 2014;1(01):18-27. Available from: 10.15419/psc.v1i01.117.

12. Rodriguez R, Rosu-Myles M, Aráuzo-Bravo M, Horrillo A, Pan Q, Gonzalez-Rey E, et al. Human bone marrow stromal cells lose immunosuppressive and anti-inflammatory properties upon oncogenic transformation. Stem Cell Reports. 2014;3(4):60619. 25358789. Available from: 10.1016/j.stemcr.2014.08.005.

13. Truong NC, Bui KH, Pham PV. Characterization of Senescence of Human Adipose-Derived Stem Cells After Long-Term Expansion; 2018. Available from: 10.1007/5584_2018_235.

14. Rubio D, Garcia-Castro J, Martín MC, de la Fuente R, Cigudosa JC, Lloyd AC, et al. Spontaneous human adult stem cell transformation. Cancer Res. 2005;65(8):3035-9. 15833829. Available from: 10.1158/0008-5472.CAN-04-4194.

15. Bernardo ME, Zaffaroni N, Novara F, Cometa AM, Avanzini MA, Moretta A, et al. Human bone marrow derived mesenchymal stem cells do not undergo transformation after long-term in vitro culture and do not exhibit telomere maintenance mechanisms. Cancer Res. 2007;67(19):9142-9. 17909019. Available from: 10.1158/0008-5472.CAN-06-4690. 
16. Tarte K, Gaillard J, Lataillade JJ, Fouillard L, Becker M, Mossafa $\mathrm{H}$, et al. Clinical-grade production of human mesenchymal stromal cells: occurrence of aneuploidy without transformation. Blood. 2010;115(8):1549-53. 20032501. Available from: 10.1182/blood-2009-05-219907.

17. Wang $Y$, Zhang Z, Chi Y, Zhang Q, Xu F, Yang Z, et al. Longterm cultured mesenchymal stem cells frequently develop genomic mutations but do not undergo malignant transformation. Cell Death Dis. 2013;4(12):e950. 24309937. Available from: $10.1038 /$ cddis.2013.480.

18. Piccinato $C A$, Sertie $A L$, Torres $N$, Ferretti $M$, Antonioli $E$. High OCT4 and Low p16(INK4A) Expressions Determine In Vitro Lifespan of Mesenchymal Stem Cells. Stem Cells Int. 2015;2015:369828. 26089914. Available from: 10.1155/2015/
369828.

19. Rayess H, Wang MB, Srivatsan ES. Cellular senescence and tumor suppressor gene p16. Int J Cancer. 2012;130(8):1715-25 22025288. Available from: 10.1002/ijc.27316.

20. Jin $Y$, Kato T, Furu M, Nasu A, Kajita $Y$, Mitsui $H$, et al. Mesenchymal stem cells cultured under hypoxia escape from senescence via down-regulation of p16 and extracellular signal regulated kinase. Biochem Biophys Res Commun 2010;391(3):1471-6. 20034468. Available from: 10.1016/j. bbrc.2009.12.096.

21. Rodriguez $R$, Rubio $R$, Masip $M$, Catalina $P$, Nieto $A$, de la Cueva $T$, et al. Loss of p53 induces tumorigenesis in p21 deficient mesenchymal stem cells. Neoplasia. 2009;11(4):397407. 19308294. Available from: 10.1593/neo.81620.

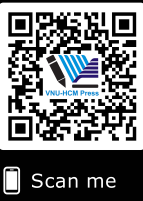

